EESTI NSV TEADUSTE AKADEEMIA TOIMETISED.

FUOSIKA * MATEMAATIKA

ИЗВЕСТИЯ АКАДЕМИИ НАУК ЭСТОНСКОИ ССР. ФИЗИКА * МАТЕМАТИКА

PROCEEDINGS OF THE ACADEMY OF SCIENCES OF THE ESTONIAN SSR.

PHYSICS * MATHEMATICS

1984, 33. 3

удК $\quad 519,2.24$

В. ОЛЬМАН

\title{
ТОЧЕЧНАЯ МИНИМАКСНАЯ ОЦЕНКА СЛУЧАЙНОЙ ВЕРОЯТНОСТИ ПРИ ОГРАНИЧЕНИЯХ НА АПРИОРНОЕ РАСПРЕДЕЛЕНИЕ
}

v. OLMAN. JUHUSLIKU TOENXOSUSE MINIMAKS PUNKTHINNANG KITSENDATUD APRIOORSE JAOTUSE KORRAL

V. OLMAN. POINT MINIMAX ESTIMATOR OF A RANDOM PROBABILITY UNDER RESTRICTIONS ON A PRIORI DISTRIBUTION

\section{(Представил Н. Алумяэ)}

Решаемая здесь задача формулируется следующим образом. Пусть $x_{1}, x_{2}, \ldots, x_{n}$ - независимые реализации биномиальной случайной величины $X$, т. е. $P(X=1)=p, P(X=0)=1-p$. Параметр $p$ тоже является случайной величиной, про распределение которой известно лишь, что оно принадлежит некоторому классу $\mathcal{F}$. Риск используемой процедуры $\delta\left(x_{1}, x_{2}, \ldots, x_{n}\right)$ при оценивании параметра $p$ имеет вид

$$
R(\delta, p)=E_{p}\left(\delta\left(x_{1}, x_{2}, \ldots, x_{n}\right)-p\right)^{2} .
$$

Задача заключается в нахождении такой $\mathcal{F}$-допустимой процедуры оценивания $\delta_{0}\left[{ }^{1}\right]$, что

$$
\inf _{\delta \in B_{\mathcal{F}^{*}}} \sup _{0 \leqslant p \leqslant 1} R(\delta, p)=\sup _{0 \leqslant p \leqslant 1} R\left(\delta_{0}, p\right),
$$

где $B_{\mathcal{F}}-$ множество всех $\mathcal{F}$-допустимых оценок, т. е. таких, для которых не существует $\delta^{*}$ со свойством $\int_{0}^{1} R(\delta, p) d F(p) \geqslant \int_{0}^{1} R\left(\delta^{*}, p\right) d F(p)$ $\forall F \in \mathcal{F} \quad$ и со строгим неравенством хотя бы для одного $F \in \mathcal{F}$. В настоящей работе эта задача решается для класса $\mathcal{F}$, описанного в $\left[{ }^{1}\right]$, т. е. $G \in \mathcal{F}$, если

1) $G(p+0)+G(1-p)=1, \quad 0 \leqslant p \leqslant 1$,

2) $G(p)$ вогнута на интервале $(0,1 / 2)$.

Как показано в $\left[{ }^{1}\right]$, класс $\mathcal{F}$-допустимых процедур совпадает с множеством байесовских оценок относительно элементов класса $\mathcal{F}$. Таким образом, с учетом того, что $y=\sum_{i=1}^{n} x_{i}$ - достаточная статистика, общий вид оценок из класса $B_{\widetilde{T}}$ следующий $\left[{ }^{2}\right]$ : 


$$
\delta_{F}(y)=\frac{\int_{0}^{1} p^{y+1}(1-p)^{n-y} d F(p)}{\int_{0}^{1} p^{y}(1-p)^{n-y} d F(p)}, \quad F \in \mathcal{F},
$$

т. е. $\delta_{F}-$ это оценка, байесовская относительно распределения $F$.

Те ор ем а. Решением задачи (1) при $n \geqslant 4$ является оценка $\delta^{*}(y)=$ $=\frac{y+1}{n+2}$, байесовская относительно равномерного на $[0,1]$ распределения $F_{0}(p)$, т. е. $F_{0}(p)=0, p \leqslant 0, F_{0}(p)=p, 0 \leqslant p \leqslant 1$.

Д ок а з ател ьство. Прямые вычисления показывают, что

$$
\begin{gathered}
R\left(\delta^{*}, p\right)=\frac{p^{2}(4-n)+p(n-4)+1}{(n+2)^{2}}, \text { и следовательно, } \\
\sup _{0 \leqslant p \leqslant 1} R\left(\delta^{*}, p\right)=R\left(\delta^{*}, 1 / 2\right) .
\end{gathered}
$$

Таким образом, для доказательства теоремы достаточно показать, что

$$
R\left(\delta_{F}, 1 / 2\right) \geqslant R\left(\delta^{*}, 1 / 2\right) \quad \forall F \in \mathcal{T} .
$$

Из вида (2) $\mathcal{F}$-допустимых оценок получаем

$$
\delta_{F}(y)=\frac{\int_{0}^{1 / 2}\left[p^{y+1}(1-p)^{n-y}+(1-p)^{y+1} p^{n-y}\right] d F(p)}{\int_{0}^{1 / 2}\left[p^{y}(1-p)^{n-y}+(1-p)^{y} p^{n-y}\right] d F(p)} .
$$

Используя это представление, легко убедиться, что для всех $F \in \mathcal{F}$ имеет место равенство

$$
\delta_{F}(y)+\delta_{F}(n-y)=1, \quad y=0,1, \ldots, n .
$$

Теперь покажем, что

$$
\delta_{F}(y) \geqslant \delta^{*}(y) \geqslant 1 / 2, \quad y \geqslant n / 2, \quad \forall F \in \mathcal{F} .
$$

То, что $\delta^{*}(y) \geqslant 1 / 2$ при $y \geqslant n / 2$ легко следует из самого вида оценки $\delta^{*}(y)$. Для доказательства левого неравенства в (6), используя формулу $(4)$, перепишем его следующим образом:

$$
\iint_{M} u_{y}(p) v_{y}(s) d F(p) d s \geqslant \iint_{M} v_{y}(p) u_{y}(s) d F(p) d s
$$

где

$$
\begin{aligned}
& \text { где } \quad M=\{(p, s): 0 \leqslant p \leqslant 1 / 2,0 \leqslant s \leqslant 1 / 2\}, u_{y}(p)= \\
& =p^{y+1}(1-p)^{n-y}+(1-p)^{y+1} p^{n-y}, \quad v_{y}(p)=p^{y}(1-p)^{n-y}+p^{n-y}(1-p)^{y} .
\end{aligned}
$$

В силу симметрии множества $M$ относительно прямой $p=s$ в неравенстве (7) можно перейти к интегрированию по множеству $M_{1 / 2}=$ $=\{(p, s): 0 \leqslant p \leqslant 1 / 2,0 \leqslant s \leqslant p\}:$

$$
\begin{aligned}
& \iint_{M_{1 / 2}}\left[u_{y}(p) v_{y}(s) d F(p) d s+u_{y}(s) v_{y}(p) d F(s) d p\right] \geqslant \\
\geqslant & \int_{M_{1 / 2}}\left[u_{y}(s) v_{y}(p) d F(p) d s+v_{y}(s) u_{y}(p) d F(s) d p\right] .
\end{aligned}
$$

Группируя подинтегральные выражения, получаем, что для доказательства (6) достаточно показать справедливость неравенства

$$
u_{y}(p) v_{y}(s)[d F(p) d s-d F(s) d p] \geqslant v_{y}(p) u_{y}(s)[d F(p) d s-d F(s) d p]
$$

для $0 \leqslant s \leqslant p \leqslant 1 / 2$. 
Выражение в квадратных скобках неположительно, так как $F \in \mathcal{F}$, и следовательно, $\frac{d F(t)}{d t}$ почти везде существует и не возрастает при $0<t<1 / 2\left[{ }^{1}\right]$. Таким образом, осталось доказать, что

$$
u_{y}(p) v_{y}(s) \leqslant v_{y}(p) u_{y}(s), \quad 0 \leqslant s \leqslant p \leqslant 1 / 2,
$$

или, что $u_{y}(p) / v_{y}(p)$ не возрастает по $p, 0 \leqslant p \leqslant 1 / 2$. Простыми преобразованиями получаем

$$
u_{y}(p) / v_{y}(p)=\frac{1}{1+t} \frac{t^{s+1}}{t^{s}+1}
$$

где $t=p /(1-p), s=2 y-n$.

Дифференцируя (10) по $p$, получаем

$$
\frac{d}{d p} \frac{u_{y}(p)}{v_{y}(p)}=\frac{1-2 p}{1-p} \frac{t^{2 s}-1+s t^{s-1}\left[t^{2}-1\right]}{\left(t^{s}+1\right)^{2}(1+t)^{2}},
$$

а. так как $p \leqslant 1 / 2$ и $y<n / 2$, то $s \geqslant 0$ и $0 \leqslant t \leqslant 1$, откуда $t^{2 s}-1 \leqslant 0$, $t^{2}-1 \leqslant 0$, и следовательно,

$$
\frac{d}{d p} \frac{u_{y}(p)}{v_{y}(p)} \leqslant 0
$$

что эквивалентно неравенству (9), а значит, и неравенству (6). Отметим, что в силу равенства (5) и неравенств (6)

$$
\delta_{F}(y) \leqslant \delta^{*}(y) \leqslant 1 / 2 \quad \text { при } y<n / 2 \quad \forall F \in \mathcal{F} .
$$

Нетрудно убедиться в том, что независпмо от четности числа $n$

$R\left(\delta_{F}, 1 / 2\right)-R\left(\delta^{*}, 1 / 2\right)=\sum_{k=0}^{\left[\frac{n-1}{2}\right]}\left[\left(\delta_{F}(k)-\frac{1}{2}\right)^{2}-\left(\delta^{*}(k)-\frac{1}{2}\right)^{2}\right] C_{n}^{k} / 2^{n}$ где $\left[\frac{n-1}{2}\right]$ - целая часть числа $\frac{n-1}{2}$, откуда имеем в силу неравенств (6)

$\left(\delta_{F}(k)-\frac{1}{2}\right)^{2}-\left(\delta^{*}(k)-\frac{1}{2}\right)^{2}=\left(\delta_{F}(k)-\delta^{*}(k)\right)\left(\delta_{F}(k)+\delta^{*}(k)-1\right) \geqslant 0$, что и доказывает неравенство (3).

\section{Л И ТЕРАТУ РА}

1. Ольман В. Изв. АН ЭССР. Физ. Матем., 33, № 3, 285-290 (1984).

2. Вальд А. Статистические решающие функции. - В кн.: Позиционные игры. М., «Наука», $1967,300-522$.

Ннститут кибернетики

Академии наук Эстонской ССР
Поступила в редакцию $26 /$ X 1983 